\title{
DISFUNCIONALIDAD FAMILIAR Y ACOSO EN ESTUDIANTES DE LA I.E. 17 DE SETIEMBRE DEL DISTRITO DEL TAMBO- HUANCAYO.
}

\section{FAMILY DYSFUNCTION AND BULLYING OF STUDENTS OF THE IE SEPTEMBER 17, DISTRICT TAMBO- HUANCAYO.}

\author{
Eugenia Fabián Arias; Nidia Matos Maldonado; Luz María Vilcas Baldeón; \\ Chinchayhuara Meléndez Carmen
}

\section{RESUMEN}

El estudio que se realizó es de nivel descriptivo correlacional, el problema es ¿Qué relación existe entre bullying y disfuncionalidad familiar en estudiantes de la Institución Educativa 17 de Setiembre del AA.HH. Juan Parra del Riego del Distrito de El Tambo?, el objetivo es determinar la relación entre bullying y disfuncionalidad familiar, la hipótesis es: Existe una relación significativa entre bullying y disfuncionalidad familiar. La población fue de 280 estudiantes, la muestra fue lo mismo de la población. Las técnicas fueron el cuestionario- escala para identificar los casos de Bullying y por otro lado para la variable disfuncionalidad familiar se utilizó el APGAR Familiar, el diseño fue no experimental descriptivo-correlacional y para demostrar la relación entre bullying y disfuncionalidad familiar se utilizó la correlación de Pearson. El resultado es la disfuncionalidad familiar es leve y el bullying se manifiesta en forma verbal en un 39\%, físico en un $30.3 \%$, social en un $22 \%$ y psicológico en un $8.6 \%$; además existe una relación directa y alta (altamente significativa) de 0.724 entre las variables de disfuncionalidad familiar y bullying verbal. La conclusión del presente estudio es que existe una relación directa y altamente significativa entre disfuncionalidad familiar y el bullying en los estudiantes de la I.E. 17 de setiembre.

Palabras Clave: Disfuncionalidad familiar, acoso físico y psicológico.

\begin{abstract}
The study conducted is descriptive correlational level, the problem is what is the relationship between bullying and family dysfunction in students of School 17 September AA.HH. Juan Parra del Irrigation District of El Tambo? the goal is to determine the relationship between bullying and family dysfunction, the hypothesis: There is a significant relationship between bullying and family dysfunction. The population was 280 students, the sample was the same population. Techniques were the scale to identify questionnaire- Bullying cases and secondly to varying dysfunctional families used the Family APGAR, design It was not experimental descriptive correlational and to demonstrate the relationship between bullying and family dysfunction Pearson's correlation was used. The result is family dysfunction is mild bullying manifests verbally by $39 \%$, physical in $30.3 \%, 22 \%$ social and psychological by $8.6 \%$; in addition there is a direct and high ratio (highly significant) of 0.724 between the variables of family dysfunction and verbal bullying. The conclusion of this study is that there is a direct and highly significant relationship between Family dysfunction and bullying among students in the IE September 17.
\end{abstract}

Keywords: Family dysfunction, bullying. 


\section{INTRODUCCIÓN}

En las últimas décadas en las instituciones educativas se han incrementado el fenómeno llamado "bullying escolar" que hace referencia, al acoso escolar y que no es otra cosa que el maltrato físico y/o psicológico deliberado y continuo" que recibe un niño o adolescente por parte de otro u otros, pudiendo traer como una consecuencia, que un alumno no quiera asistir al colegio con temor de denunciar a sus agresores.

La conciencia juvenil en instituciones educativas en los últimos años, ha sido muy difícil y complejo, esto ha repercutido en acciones que dañan a los estudiantes de nivel secundario ocasionando deterioro en susalud, a nivel emocional, como en lo físico (al recibir golpes). A esta situación se le debe agregar el componente familiar, así la importancia de la familia en la formación de la personalidad es determinante, ya que es el primer elemento de socialización del niño y las investigaciones afirman que los modelos de familias pueden ser predictores de conductas violentas en los niños y adolescentes. En este sentido, se investigó la disfuncionalidad familiar y el bullying en la I.E. 17 de setiembre del AA.HH. Juan Parra del Riego del Distrito del Tambo de Huancayo.

\section{MATERIAL Y MÉTODOS}

El presente estudio es de tipo Básico, el nivel es descriptivo en la parte inicial luego correlacional, el método de investigación fue el analítico - sintético, la población fue de 280 alumnos aproximadamente y la muestra fue el mismo de la población que afrontan el problema del bullying (que fue identificado en un numero de 23 estudiantes) por lo que para la variable disfuncionalidad familiar se aplicó el APGAR familiar solo para los 23 padres de familia de los estudiantes que afrontan el bullying, así mismo los instrumentos a utilizados fueron el cuestionarioescala, la guía de entrevista y el APGAR familiar.

Para establecer la relación entre bullying y disfuncionalidad familiar se utilizó la correlación de Pearson.

\section{RESULTADOS Y DISCUSIÓN}

\section{RESPECTO A LA HIPÓTESIS ESPECÍFICA I}

La disfuncionalidad familiar en los alumnos de la I.E. 17 de setiembre del AA.HH. Juan Parra del Riego del
Distrito del Tambo - Huancayo es leve, los resultados logrados fueron:

DISFUNCIONALIDAD FAMILIAR.- De acuerdo al instrumento (APGAR FAMILIAR) aplicado en la investigación fue:

\section{CUADRO No 1}

Funcionalidad familiar de los alumnos de la Institución Educativa 17 de Setiembre del AA.HH. Juan Parra Del Riego

\begin{tabular}{|l|c|c|c|c|c|}
\hline \multicolumn{1}{|c|}{ Función } & nunca & $\begin{array}{c}\text { casi } \\
\text { nunca }\end{array}$ & $\begin{array}{c}\text { algunas } \\
\text { veces }\end{array}$ & $\begin{array}{c}\text { casi } \\
\text { siempre }\end{array}$ & siempre \\
\hline $\begin{array}{l}\text { Me satisface la ayuda } \\
\text { que recibo de mi } \\
\text { familia cuando tengo } \\
\text { algún problema y/0 } \\
\text { necesidad. }\end{array}$ & 2 & 2 & 3 & 2 & 3 \\
\hline $\begin{array}{l}\text { Me satisface la partici- } \\
\text { pación que mi familia } \\
\text { me brinda y permite }\end{array}$ & 3 & 4 & 2 & 2 & 1 \\
\hline $\begin{array}{l}\text { Me satisface cómo mi } \\
\text { familia acepta y apoya } \\
\text { mis deseos de empren- } \\
\text { der nuevas actividades. }\end{array}$ & 1 & 2 & 3 & 4 & 2 \\
\hline $\begin{array}{l}\text { Me satisface cómo mi } \\
\text { familia expresa afectos } \\
\text { y responde a mis } \\
\text { emociones como rabia, } \\
\text { tristeza, amor y otros. }\end{array}$ & 4 & 2 & 2 & 1 & 3 \\
\hline $\begin{array}{l}\text { Me satisface cómo } \\
\text { compartimos en mi } \\
\text { familia: a) el tiempo } \\
\text { para estar juntos, b) los } \\
\text { espacios en la casa, c) } \\
\text { el dinero. }\end{array}$ & 2 & 2 & 3 & 2 & 3 \\
\hline $\begin{array}{l}\text { Estoy satisfecha con el } \\
\text { soporte que recibo que } \\
\text { recibo de mis amigos } \\
\text { (as). }\end{array}$ & 3 & 2 & 2 & 3 & 3 \\
\hline $\begin{array}{l}\text { ¿Tiene usted algún (a) } \\
\text { amigo (a) cercano (a) } \\
\text { a quién pueda buscar } \\
\text { cuando necesita ayuda? }\end{array}$ & 2 & 2 & 17 & \\
\hline & 17 & 16 & & \\
\hline
\end{tabular}

Considerando que la Escala para la calificación de la Funcionalidad de la familia de acuerdo al APGAR FAMILIAR es:

\begin{tabular}{|l|c|c|}
\hline \multicolumn{1}{|c|}{ NIVELES DE FUNCIONALIDAD } & PUNTAJE & VALORACIÓN \\
\hline Buena Función Familiar & $18-20$ & Alta. \\
\hline Disfunción Familiar Leve & $14-17$ & Media \\
\hline Disfunción familiar moderada & $10-13$ & Media \\
\hline Disfunción Familiar Severa & 9 ó menos & Muy baja \\
\hline
\end{tabular}


De acuerdo al puntaje obtenido de las respuestas, los alumnos victimarios proceden de familias que tienen disfuncionalidad familiar leve, porque los puntajes obtenidos en el cuadro oscilan de acuerdo a la valoración de 14 a 17 que es considerado media.

El estudio realizado sobre "Funcionalidad familiar y acoso escolar o bullying en adolescentes del Distrito de El Alto, Piura, Perú", al realizar la investigación encontró que alrededor del $25 \%$ de los acosadores (Buller) presentan desestructuración familiar, malos métodos de crianza, malos tratos y falta de afecto entre cónyugues y violencia familiar. Por su lado, el riesgo de ser víctima se asocia a malasprácticas de crianza como la sobre protección, no tener como núcleo de convivencia al padre 0 a la madre, padres no unidos, bajo nivel educativo de los padres y no prestar atención a la queja de los hijos.Asimismo se sustenta enCamacho, León , Silva (2009), en el estudio del "Funcionamiento familiar", según el modelo Circunflejo de Olson en adolescentes de la I.E. No 2053 "Francisco Bolognesi Cervantes" del Distrito de Independencia - Lima, encontraron de una población de 223 adolescentes, más de la mitad (54.71\%) representa a la familia con un funcionamiento familiar no saludable y con tendencia a la disfuncionalidad, con extrema separación emocional y poco involucramiento entre sus miembros, siendo que los roles de cada miembro no son claros, causando frecuentes cambios en las reglas y las decisiones parentales. Además se sustenta en Moreno, Vacas y Roa (2006) en el estudio sobre Victimización escolar y clima socio-familiar. Encontrando que el 24.65\% era victimizado. Los niños menores de 12 años eran los más victimizadores y victimizados. Además encontraron que cuando la vida familiar se rige por estrechas reglas, 0 las familias son conflictivas, el trato percibido, tanto de la familia como en la escuela, por los niños y adolescentes, es deficiente, además de aumentar la probabilidad de comportamientos sociales desadaptados por parte de los hijos. Cuando en la familia existen, por el contrario, vías para la expresión de los sentimientos y se anima a que sus miembros actúen libremente, cuando los intereses culturales, así como los sociales y recreativos son cultivados, cuando se organizan las responsabilidades familiares y cuando hay cohesión, es decir, ayuda, compenetración y apoyo entre los miembros, la probabilidad de comportamientos victimizadores, 0 sufrir mal trato en la escuela y en la casa, es baja o nula. Finalmente se sustenta en Estévez, Murgui, Moreno, y Musito (2007)en el estudio sobre estilos de comunicación familiar, actitud hacia la autoridad institucional y conducta violenta del adolescente en la escuela, en una muestra de 1,049 escolares de ambos sexos. Encontraron que existe una estrecha asociación entre la comunicación negativa con el padre y la conducta violenta en la adolescencia. También encontraron que existe una influencia indirecta del padre, la madre y el profesor en el comportamiento violento del hijo-alumno, fundamentalmente a través de su efecto en el autoconcepto familiar y escolar.

\section{HIPÓTESIS ESPECIFICA II}

El bullying en los alumnos de la I.E. 17 de setiembre del distrito del Tambo - Hyo, se presenta con conductas agresivas, insultos, intimidaciones, burlas y diversas formas de acoso.

Cuadro $\mathrm{N}^{\circ}$ 2: Frecuencia de maltrato escolar 0 bullying

\begin{tabular}{|l|l|c|c|c|c|}
\hline \multicolumn{2}{|c|}{$\begin{array}{l}\text { Cuantas veces ha sufrido maltra- } \\
\text { to de parte de sus compañeros. }\end{array}$} & SI & $\%$ & N0 & $\%$ \\
\hline 1 & $\begin{array}{l}\text { No he sido nunca maltratado } \\
\text { o intimidado por mis com- } \\
\text { pañeros }\end{array}$ & - & - & 62 & $22.14 \%$ \\
\hline 2 & $\begin{array}{l}\text { Solo sucedió una vez por } \\
\text { año }\end{array}$ & 104 & $37.14 \%$ & & \\
\hline 3 & Sucedió 2 veces al año & 62 & $22.14 \%$ & & \\
\hline 4 & Sucedió 1 vez al mes & 29 & $10.35 \%$ & & \\
\hline 5 & $\begin{array}{l}\text { Sucedió varias veces a la } \\
\text { semana }\end{array}$ & 23 & $8.21 \%$ & & \\
\hline & SUB TOTAL & 218 & & 62 & \\
\hline & TOTAL & \multicolumn{2}{|c|}{280} & & \\
\hline
\end{tabular}

Fuente: Cuestionario aplicado por las investigadoras a los estudiantes de la I.E. 17 DE SETIEMBRE de AA.HH. Juan Parra del Riego.- Junio del 2012

En el cuadro se aprecia que el $22.14 \%$ manifiestan no haber sido nunca maltratados 0 intimidados por sus compañeros, el $37.14 \%$ respondieron que fueron maltratados solo una vez por año, así mismo un 
22.14\% manifiesta haber sido maltratado 2 veces al año, también hacen mención un 10.35\% haber sido maltratado 1 veces al mes y un mínimo porcentaje que es el $8.21 \%$ sucedió varias veces a la semana, en este sentido de acuerdo a la literatura sobre el tema del bullyng tendríamos 23 estudiantes que afrontan el problema del bullyng.

\section{Cuadro $\mathrm{N}^{\circ} 3$}

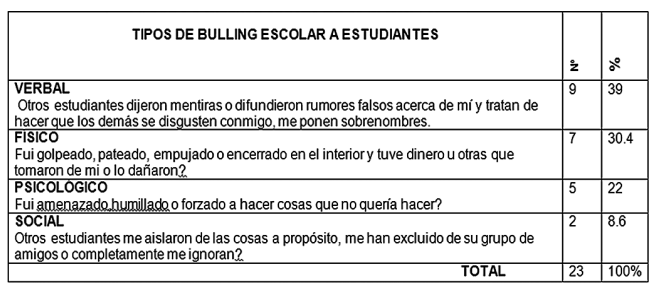

Fuente: Guía de entrevista aplicado por las investigadoras a los estudiantes de la I.E. 17 DE SETIEMBRE de AA.HH. Juan Parra del Riego. 12 de Junio del 2012

El Bullyng se manifiesta a través del maltrato verbal en un $39 \%$, físico en un $30.4 \%$, social en un $8.6 \%$ y psicológico 22\%.

\section{Cuadro $N^{\circ} 4$}

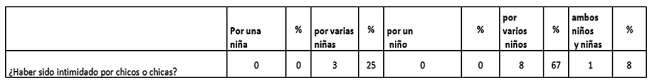

Fuente: Guía de entrevista aplicado por las investigadoras a los estudiantes de la I.E. 17 DE SETIEMBRE de AA.HH. Juan Parra del Riego. 12 de Junio del 2012

Los victimarios o intimidadores es más practicado por niños $67 \%$, niñas 33\%. Así mismo el número de alumnos que intimidan fue de 4 a 9 estudiantes que hacen un 58\%, el tiempo de la intimidación es de un año que equivale un 58\%, los lugares donde han sido intimidados fueron a la salida del colegio $21.8 \%$, así mismo en las horas del recreo el $21.8 \%$ y seguido de la salida del colegio, en el baño y en clase sin la presencia del profesor. Estos resultados se sustentan en Cisneros X, Oñate, (2007) en el trabajo de investigación que realizó a una muestra de 24.990 alumnos de 14 comunidades autónomas, encontró que el $13.90 \%$ de los alumnos es agredido por sobrenombres, el $10.40 \%$ por el silencio o no dirigirles la palabra. Se encontró también que la tasa de acoso entre niños es de $24.4 \%$, la tasa de acoso entre las niñas fue de $21.6 \%$ y la tasa de acoso total fue de 23.3\%. Así mismo se sustenta en Castañeda (2003, en García y Madríaza, 2005) en el estudio realizado demostró que el $28 \%$ de los estudiantes reconoce haber participado en peleas, un $71 \%$ dice haber sido agredido verbalmente y un $18 \%$ dice haber visto a un compañero portando armas. Por otra parte el $26 \%$ de los niños de 6 y 10 años reconoce haber participado en peleas. Por otra parte, se sustenta en Oliveros y Barrientos (2007)en el estudio sobre incidencia y factores de riesgo de la intimidación (bullying) en un colegio particular de Lima Metropolitana con una muestra de 185 alumnos. Se determinó que un 54.7\% de ellos habían sufrido bullying. El 52.7\% fueron mujeres y el $47.3 \%$ varones. El $78.4 \%$ vive con ambos padres. La intimidación verbal había predominado con $38.7 \%$ de incidencia y el $84.3 \%$ de alumnos no habían defendido a sus compañeros. Además, se sustenta en DEVIDA (2007) en la investigación efectuada a nivel nacional, reportaron que el $40 \%$ del total de escolares secundarios son víctimas de agresiones en las modalidades de ignorados (28\%), excluidos (22\%), discriminados (21\%), físicamente agredidos (24\%) y sexualmente acosados (10\%). Así mismo se sustenta en Oliveros, Figueroa, Mayorga, Cano, Quispe y Barrientos (2009) en el estudio realizado sobre la Intimidación en colegios estatales de secundaria del Perú. Presentaron las siguientes evidencias, como que la incidencia de intimidación tuvo un promedio de 50,7\%. Las variables asociadas fueron apodos, golpes, falta de comunicación........ También se sustenta en Becerra et al (2009)En una muestra de 1087 estudiantes secundarios de Lima Metropolitana encontraron que el $45 \%$ de la muestra había sido víctima de agresión, la modalidad más frecuente era poniendo sobrenombres $69 \%$. Y finalmente se sustenta en Peña (2009) en el estudio realizado sobre "Bullying, Problema latente en los colegios"; hace referencia lo siguiente:

El 10\% de escolares de colegios de San Juan de Lurigancho, Centrode Lima y parte del Cono Sur sufren de bullying. Las modalidades utilizadas van desde la intimidación física hasta la intimidación psicológica expresada en amenazas, humillación, insultos, apodos 0 
exclusión; la intimidación psicológica es la más utilizada porque no deja huellas visibles como la agresión física, sin embargo sus efectos persisten a lo largo del tiempo.

\section{HIPÓTESIS GENERAL}

Si existe relación significativa entre disfuncionalidad familiar y el Bullying en los alumnos de la I.E. 17 de setiembre del AA.HH. Juan Parra del Riego del Distrito del Tambo, porque existe autoritarismo, negligencia y falta de disciplina.

\section{RELACIÓN ENTRE DISFUNCIONALIDAD FAMILIAR Y LA VARIABLE BULLYING.}

Para la correlación se utilizó una muestra de 280alumnos, los resultados de los estadísticos descriptivos se muestran en la Tabla 1.

Tabla 1. Estadísticos descriptivos

\begin{tabular}{lccc}
\hline & Media & Desviación típica & N \\
\hline Disfuncionalidad Familiar & 14.6036 & .84029 & 280 \\
Bullying & 3.4036 & .93017 & 280 \\
\hline
\end{tabular}

Así mismo los resultados de la correlación entre estas dos variables se muestran en la Tabla 2.

Tabla № 2 Correlación

\begin{tabular}{llcc}
\hline & & $\begin{array}{c}\text { Disfuncionalidad } \\
\text { Familiar }\end{array}$ & Bullying \\
\hline $\begin{array}{l}\text { Disfuncionabilidad } \\
\text { Familiar }\end{array}$ & $\begin{array}{l}\text { Correlación de } \\
\text { Pearson }\end{array}$ & 1 & $.724\left(^{* *}\right)$ \\
& Sig. (bilateral) & & .000 \\
Bullying & $\mathrm{N}$ & 280 & 280 \\
& Correlación de & $.724\left(^{* *}\right)$ & 1 \\
& Pearson & .000 & \\
& Sig. (bilateral) & 280 & 280 \\
\hline
\end{tabular}

** La correlación es significativa al nivel 0,01 (bilateral).

En la tabla № 2 se observa que el $r$ (Coeficiente de Correlación de Pearson) es igual a $0.724^{\star \star}$, este valor nos señala que existe una relación alta (altamente significativa) entre las variables disfuncionalidad familiar y el Bullying. Además rtiene valor positivo indicando que la correlación es directa o positiva. Corresponde a una $p$ de 0.000 , la que es menor que 0.05 por lo que se rechaza la hipótesis nula $\left(\mathrm{H}_{0}\right)$ aceptando la hipótesis planteada $\left(\mathrm{H}_{1}\right)$.
Estos resultados se sustentan en Santander, Zubarew, Santelices, Argollo, Cerda y Bórquez (2008) en un estudio sobre la influencia de la familia como factor protector de conductas de riesgo en escolares chilenos, hallaron que el $66.5 \%$ de los estudiantes percibe que sus familias son disfuncionales. Casi la mitad de los escolares implicados en este estudio manifiesta que sus padres no dedican el tiempo adecuado para conversar, además, refieren falta de conocimiento de las actividades de sus hijos, falta de reglas claras en la casa, ausencia de rituales familiares y de actividades conjuntas. Todo lo cual podría estar relacionado con el nivel sociocultural de las familias y el cambio que ha sucedido en las familias chilenas, que impiden una conexión adecuada con los hijos, lo que sumado a distorsiones en las representaciones mentales acerca de la tarea educativa del padre y la madre determinaría la presencia de mayores conductas de riesgo en escolares .Así mismo se sustenta en Yuste, y Pérez (2008) En el estudio sobre las cuestiones familiares como posible causa de la violencia escolar, hallaron que los padres destacan como aspectos que más influyen en el origen o génesis de las conductas violentas en la escuela son: la escasa educación en el respeto a los demás y a las cosas y la falta de educación en los valores. Además se sustenta en González y de la Hoz (2011), en la investigación realizada sobre "Relaciones entre los comportamientos de riesgo psicosociales y la familia en adolescentes de Suba, Bogotá". En el Estudio observacional de corte transversal entre 12302 adolescentes (10 a 19 años de edad), estudiantes de 12 colegios públicos de la localidad de Suba en el año 2006. Se aplicó una encuesta auto diligenciada donde se interrogó por comportamientos sexuales, adictivos, depresivos, violentos y de trastornos de conducta alimentaria.

Los resultados fueron: Se encontró una prevalencia de buena función familiar de 63 \% y de disfunción familiar severa del $7 \%$. La disfunción severa aumentó la prevalencia de comportamientos violentos (OR de $4,1$ con IC de $95 \% 3,4-4,7)$, trastornos de conducta alimentaria (OR 2,3 con IC de $95 \%$ de 1,9-2,8) y relaciones sexuales (OR de 2 con IC de $95 \%$ de 1,72,4). Por el contrario, la composición de la familia (presencia o no de ambos padres) no mostró relación 
con comportamientos violentos.

Las conclusiones fueron: La disfunción familiar se asocia a una mayor frecuencia de conductas de riesgo en los adolescentes de bajo estrato socioeconómico en Bogotá. La relación más fuerte se observa entre violencia y familia.

\section{CONCLUSIONES}

1. La disfuncionalidad familiar en los alumnos de la I.E. 17 de Setiembre del distrito del Tambo es leve porque de acuerdo a la valoración de la escala del APGAR FAMILIAR el puntaje obtenido de las respuestas oscila de 14 a 17 es leve, porque de acuerdo a la escala de valoración es considerado media.

2. El Bullyng se manifiesta a través del maltrato verbal en un 39\%, físico en un 30\%, social en un $22 \%$ y psicológico 6\%. Los victimarios o intimidadores es más practicado por niños $67 \%$, niñas $33 \%$. Así mismo el número de alumnos que intimidan fue de 4 a 9 estudiantes que hacen un 58\%, el tiempo de la intimidación es de un año que equivale un 58\%, los lugares donde han sido intimidados fueron a la salida del colegio 21.8\%, así mismo en las horas del recreo el $21.8 \%$ y seguido de la salida del colegio, en el baño y en clase sin la presencia del profesor.

3. La relación que existe en la disfuncionalidad familiar y bullyng en los estudiantes de la I.E. 17 de setiembre es altamente significativo porque_el Coeficiente de Correlación de Pearson es igual a $0.724^{\star \star}$, este valor nos señala que existe una relación alta (altamente significativa) entre las variables disfuncionalidad familiar y el Bullying. Además rtiene valor positivo indicando que la correlación es directa o positiva.

\section{REFERENCIAS BIBLIOGRÁFICAS:}

1. A Bramorag. M (2003) Enfrentando la violencia en las escuelas. Un informe de Brasil. Violencia en las escuelas, América Latina y el Caribe, Brasilia; UNESCO.

2. Avilés, J.M. (2003) Bullying: intimidación y maltrato entre el alumnado. Bilbao.

3. Cerezo, F. (2007) La violencia en las aulas, $5^{\text {ta }}$ edición, Editorial Pirámide, Madrid - España.

4. Comisión Nacional para el Desarrollo y Vida sin Drogas: DE VIDA (2007), Estudio Nacional: Prevención y consumo de drogas en estudiantes secundarios, recuperado de : http://www.devida. gob.pe/documentacion/documentos disponibles/ll Estudio Regional Escolar Sección 2007.pdf

5. Movimiento Manuela Ramos, (2010) Violencia Familiar, recuperado de: http://www.manuela.org.pe/ violencia.asp.

6. Piñuel, I- Oñate, A.(2007). Mobbing Escolar: Violencia y acoso psicológico contra los niños. Barcelona, CEAC.

7. Serrano, A, (2006). Acoso y Violencia en la Escuela, Editorial Ariel. Barcelona- España.

8. Ccoicca, T. (2010) "Bulling y Funcionalidad Familiar en una Institución Educativa del Distrito de Comas" Lima Perú.

\section{INSTRUCCIONES PARA LOS AUTORES}

La revista "Prospectiva Universitaria" es una revista científica publicado por el Centro de Investigación de la Universidad Nacional del Centro del Perú; integra las investigaciones en varias áreas de la ciencia, desarrollados por investigadores de la UNCP; podrían ser:

1. Artículos Científicos Originales

2. Artículos o notas científicas.

3. Artículos de Revisión

4. Cartas al editor. 